\title{
Noise-induced escape on time scales preceding quasistationarity: New developments in the Kramers problem
}

\author{
S. M. Soskin, V. I. Sheka, and T. L. Linnik \\ Institute of Semiconductor Physics, National Academy of Sciences of Ukraine, Kiev, Ukraine \\ M. Arrayás \\ Department of Physics, Lancaster University, Lancaster, LA1 4YB, United Kingdom \\ and Instituut-Lorentz, Universiteit Leiden, Postbus 9506, 2300 RA Leiden, The Netherlands \\ I. Kh. Kaufman and D. G. Luchinsky \\ Department of Physics, Lancaster University, Lancaster, LA1 4YB, United Kingdom \\ and Russian Research Institute for Metrological Service, Ozernaya 46, 119361 Moscow, Russia \\ P. V. E. McClintock ${ }^{\text {a) }}$ \\ Department of Physics, Lancaster University, Lancaster, LA1 4YB, United Kingdom \\ R. Mannella \\ Department of Physics, Lancaster University, Lancaster, LA1 4YB, United Kingdom \\ and Dipartimento di Fisica, Università di Pisa and INFM UdR Pisa, 56100 Pisa, Italy
}

(Received 28 November 2000; accepted 11 April 2001; published 31 August 2001)

Noise-induced escape from the metastable part of a potential is considered on time scales preceding the formation of quasiequilibrium within that part of the potential. It is shown that, counterintuitively, the escape flux may then depend exponentially strongly, and in a complicated manner, on time and friction. (C) 2001 American Institute of Physics. [DOI: 10.1063/1.1378788]

The problem of noise-induced escape from a metastable state of a dynamical system is of great importance in many applications. For a potential system, it is known as the Kramers problem, in allusion to the famous paper by Kramers. Until very recently, there remained a gap in the theory of the Kramers problem: How does the escape flux evolve from zero, at the initial instant, to the quasistationary stage after quasiequilibrium has been formed within the metastable state? Intuition suggests that the evolution should be monotonic and without any irregularities, which appears to be confirmed by the rigorous analysis of some partial regimes. However, as we show in the following, either the flux itself (multiwell case) or its derivative (single-well case) may evolve in a markedly nonmonotonic fashion. This nonmonotonicity, like other irregularities, relates to characteristic changes in the topology of the most probable escape path. In the multiwell case, the formation of quasiequilibrium takes an exponentially long time, so that our results are relevant to a major portion of the time attainable at small noise intensities. In the single-well case, our results are relevant to much shorter time scales (of the order of a period of eigenoscillation); interest in such short time scales is of course growing fast, as modern technologies develop.

\section{INTRODUCTION}

In his seminal work, ${ }^{1}$ Kramers considered a weak noiseinduced flux from a single metastable classical potential well, i.e., he considered a stochastic system

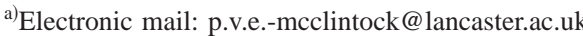

$$
\begin{aligned}
& \ddot{q}+\Gamma \dot{q}+d U / d q=f(t), \\
& \langle f(t)\rangle=0, \quad\left\langle f(t) f\left(t^{\prime}\right)\right\rangle=2 \Gamma T \delta\left(t-t^{\prime}\right), \quad T \ll \Delta U,
\end{aligned}
$$

which was put initially at the bottom of a metastable potential well $U(q)$ with a barrier $\Delta U$ and he then calculated the quasistationary probability flux across the barrier. Models of type (1) are relevant to chemical reactions, ${ }^{1}$ superconducting quantum interference devices (SQUIDs), ${ }^{2}$ and many other real systems ${ }^{3,4}$ including the recently designed mechanical electrometers. ${ }^{5}$

There have been many developments and generalizations of the Kramers problem (see Refs. 3 and 4 for reviews) but both Kramers and most of those who followed him considered only the quasistationary flux, i.e., the flux established after the formation of a quasiequilibrium distribution within the well (up to the barrier). The quasistationary flux is characterized by a slow exponential decay in time $t$, an Arrhenius dependence on temperature $T$, and a relatively weak dependence on friction $\Gamma$ :

$$
J_{\mathrm{qs}}(t)=\alpha_{\mathrm{qs}} e^{-\alpha_{\mathrm{qs}} t}, \quad \alpha_{\mathrm{qs}}=P e^{-\Delta U / T},
$$

where $P$ depends on $\Gamma$ and $T$ in a nonactivated way.

But how does the flux evolve from its zero value at initial time to its quasistationary regime (2) at time scales exceeding the time $t_{f}$ for the formation of quasiequilibrium? The answer may obviously depend on initial conditions and a relevant boundary (i.e., the boundary through which the escape occurs). As for the boundary, it can be shown that the most general qualitative features of the flux are valid for any type of boundary (for the sake of simplicity, we shall consider in the following only the absorbing wall). As for the initial conditions, their relevance may vary. The simplest and 
often relevant initial state is the bottom of the well, since it is the stable stationary state in the absence of noise: If the noise (not necessarily of the thermal origin) is switched on at some instant, then the time evolution of the escape from the bottom becomes relevant. It should be emphasized however that, if the relevant metastable part of the potential is multiwell, then the flux during the major part of the relevant time is not sensitive to the initial state provided it is concentrated just in one well (e.g., it may be thermalized in the well). As for the single-well case, the flux evolution is more sensitive to the initial state and we shall consider various cases. But, first, let us discuss the most simple case where the initial state is at the bottom of the potential. We shall refer to it as the bottom initial state.

It may seem natural to assume that the flux evolution from zero to the quasistationary regime is a monotonic function without any "irregularities." Apart from the naive argument that "noise smooths everything," this assumption appears sound because the probability distribution $W$ is distinctly centered at the bottom of the well both initially and in the quasistationary stage: $W\left(q, \dot{q}, t=0 \mid q_{0}=q_{b}, \dot{q}_{0}=0\right)$ $=\delta\left(q-q_{b}\right) \delta(\dot{q})$ while $W\left(q, \dot{q}, t \gg t_{f} \mid q_{0}=q_{b}, \dot{q}_{0}=0\right)$ is a narrow peak of width $\propto \sqrt{T}$ around that same state $\{q$ $\left.=q_{b}, \dot{q}=0\right\}$. Moreover, it was shown recently ${ }^{6}$ that, both in the underdamped and overdamped limits, the escape flux $J(t)$ does grow at $t \sim t_{f}$ in a simple manner.

Despite the above-mentioned arguments, it can be shown that, generically, $J$ evolves from $J(0)=0$ to $J_{\mathrm{qs}}\left(t \gg t_{f}\right)$ in a quite complicated way.

(1) As shown in Sec. II, the flux grows stepwise on time scales of the order of a period of eigenoscillation in the bottom of the well. Apart from filling the "gap" in time scales in the Kramers problem (cf. the big activity in the 1980s aimed at filling the gap in the friction ranges ${ }^{3,4}$ ), this part of our work is motivated by the growing interest in the very short time scales that are now relevant to certain experiments, such as those studying chemical reactions down to femtosecond time scales: ${ }^{7}$ The period of eigenoscillations relevant to chemical reactions in Ref. 7 is $\sim 1-100$ fs.

(2) As shown below in Sec. III, the evolution of the flux on longer time scales in a multiwell metastable potential is also distinctly different from the relatively simple monotonic function described in Ref. 6: $J$ grows sharply on a logarithmic time scale to a value which is typically very different from $J_{\mathrm{qs}}(0)$ (typically, exponentially larger) and then evolves to $J_{\mathrm{qs}}(t)$ during the exponentially long time.

It should be emphasized that the qualitative features of $J(t)$ described previously are valid for any reasonable definition of the flux, e.g., the full flux through a boundary or just the first-passage flux, while the boundary may be a given coordinate, or a boundary of a basin of attraction, or a boundary of the vicinity of another attractor, etc.

To illustrate our results, we will use the potential

$$
U(q)=q-q^{3} / 3
$$

for the single-well case (Fig. 1), and

$$
U(q)=0.06(q+1.5)^{2}-\cos (q)
$$

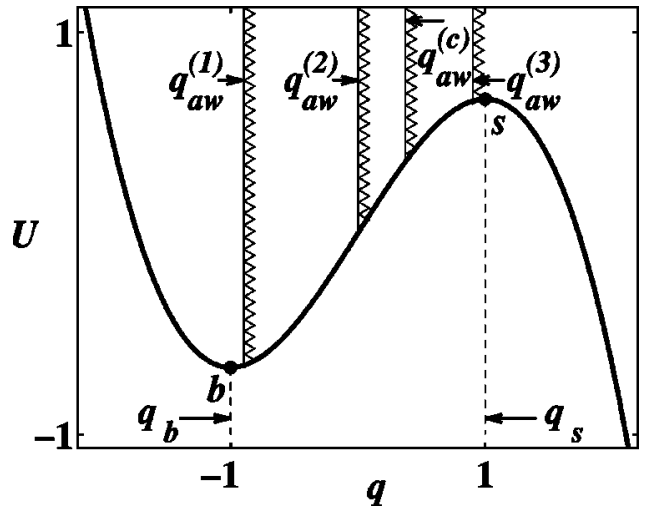

FIG. 1. The potential $U(q)=q-q^{3} / 3$. The bottom and the saddle are marked as $b$ and $s$, respectively. Triangles indicate four typical positions of the absorbing wall.

for the multiwell case [Fig. 6(a)], with an absorbing wall ${ }^{8}$ at $q=q_{\text {aw }}$ in both cases.

Experimentally, the flux is measured in the following way. The system is placed at an initial state, after which it follows the stochastic equation (1) until either the coordinate of the wall, $q_{\mathrm{aw}}$, or the time limit, ${ }^{9} t_{l}$, is reached. It is then reset to the initial state and everything is repeated. Once the statistics are deemed adequate, we calculate the flux

$$
J(t) \equiv \frac{1}{N_{\text {reset }}} \frac{\Delta N(t)}{\Delta t},
$$

where $N_{\text {reset }}$ is the overall number of resets, and $\Delta N(t)$ is the number of resets during the interval $[t, t+\Delta t] ; \Delta t$ is chosen to be much smaller than a characteristic time over which the flux (5) may change significantly, but large enough to provide $\Delta N(t) \gg 1$ (roughly, the latter is satisfied provided $\Delta t$ $\left.\gg t_{l} / N_{\text {reset }}\right)$.

The above-given experimental definition corresponds to the following theoretical definition of the flux:

$$
\begin{aligned}
& J(t)=\iint d q_{0} d \dot{q}_{0} W_{\mathrm{in}}\left(q_{0}, \dot{q}_{0}\right) J_{q_{0}, \dot{q}_{0}}(t), \\
& J_{q_{0}, \dot{q}_{0}}(t)=\int_{0}^{\infty} d \dot{q} \dot{q} W\left(q=q_{\mathrm{aw}}, \dot{q}, t \mid q_{0}, \dot{q}_{0}\right),
\end{aligned}
$$

where $W_{\text {in }}\left(q_{0}, \dot{q}_{0}\right)$ is a statistical distribution of the initial coordinate and velocity and $W$ is the conditional probability density.

The theoretical approach which we use is the method of optimal fluctuation (e.g., Refs. 10 and 11) whose details in application to the present problems are given in the following. The theoretical results are verified by computer and ana$\log$ electronic simulations. A preliminary report and discussion of some of the central ideas was given in Refs. 12 and 13.

\section{SINGLE-WELL METASTABLE POTENTIAL}

It can be shown directly from the Fokker-Planck equation that the formation of quasiequilibrium up to the barrier in the single metastable well typically takes ${ }^{14,15}$ a time of the order of 


$$
t_{f}^{(s)} \sim \frac{1}{\min \left(\Gamma, \omega_{0}^{2} / \Gamma\right)} \ln \left(\frac{\Delta U}{T}\right),
$$

where $\omega_{0}$ is the frequency of eigenoscillation in the bottom of the well.

In this section, we shall be interested in much smaller time scales,

$$
t \ll t_{f}^{(s)} .
$$

The previous work on nonstationary escape rates in the Kramers problem was based on the direct solution of the Fokker-Planck equation (cf. Ref. 6). We apply the method of optimal fluctuation to this problem for the first time, ${ }^{16-20}$ obtaining nontrivial new results for short time scales. It is convenient to consider first the case of an initial state with a given coordinate and velocity:

$$
W_{\text {in }}\left(q_{0}, \dot{q}_{0}\right)=\delta\left(q_{0}-q_{i}\right) \delta\left(\dot{q}_{0}-\dot{q}_{i}\right) .
$$

Then the flux is sought as

$$
J(t) \equiv J_{q_{i}}, q_{i}(t)=P(t) \exp \left(-\frac{S_{\text {min }}(t)}{T}\right)
$$

where the activation energy $S_{\min }(t)$ does not depend on $T$ while the prefactor $P(t)$ depends on $T$ in a nonactivated way. At small $T$ and short $t$, the factor $\exp \left(-S_{\min } / T\right)$ depends on $t$ much more strongly than $P$. So, we concentrate on studying $S_{\min }(t)$, which can be shown ${ }^{21}$ to be a minimum of the functional:

$$
\begin{aligned}
& S_{\min }(t) \equiv S_{\min }\left(q_{i}, \dot{q}_{i}, t\right)=\min _{[q(\tau)], \dot{q}(t)}(S), \\
& S \equiv S_{\dot{q}(t)}[q(\tau)]=\int_{0}^{t} d \tau L, \\
& L=(\ddot{q}+\Gamma \dot{q}+d U / d q)^{2} /(4 \Gamma), \\
& q(0)=q_{i}, \quad \dot{q}(0)=\dot{q}_{i}, \quad q(t)=q_{\mathrm{aw}} .
\end{aligned}
$$

The minimization is done over an escape path $[q(\tau)]$ at a given exit velocity $\dot{q}(t)$, with a further minimization over this velocity (a minimization similar to the latter was used in a different context in Ref. 20). Note that the minimization over the exit velocity (or, more generally, over states on a relevant boundary) was not used in applications of the method of optimal fluctuation to quasistationary escape rates $^{21,22}$ or related quantities ${ }^{23}$ since the exit occurred necessarily through the saddle. The path minimizing $S$ may be called the most probable escape path (MPEP), in analogy with the quasistationary case. The necessary conditions for the minimum of the functional (11) are as follows.

(1) A zero variation, $\delta S=0$ : It implies that the MPEP $[q(\tau)]$ satisfies the Euler-Poisson equation ${ }^{24,21}$

$$
\frac{\partial L}{\partial q}-\frac{d}{d t}\left(\frac{\partial L}{\partial \dot{q}}\right)+\frac{d^{2}}{d t^{2}}\left(\frac{\partial L}{\partial \ddot{q}}\right)=0,
$$

which, for the $L$ of the form (12), reads

$$
\dddot{q}+\ddot{q}\left(2 \frac{d^{2} U}{d q^{2}}-\Gamma^{2}\right)+\dot{q}^{2} \frac{d^{3} U}{d q^{3}}+\frac{d^{2} U}{d q^{2}} \frac{d U}{d q}=0 .
$$

(2) A zero derivative with respect to the exit velocity, $\partial S / \partial \dot{q}(t)=0$ : This condition can be reduced to $\partial L / \partial \ddot{q}(t)$ $=0$, which, for the $L$ of the form (12), reads

$$
\left.[\ddot{q}+\Gamma \dot{q}+d U / d q]\right|_{\tau=t}=0 .
$$

Solutions of Eq. (15) satisfying conditions (13) and (16) can be found numerically: In addition to $q(0)$ and $\dot{q}(0)$ given in (13), one can match $\ddot{q}(0)$ and $\ddot{q}(0)$ so that the result of the integration (15) on the interval $[0, t]$ satisfies the last of conditions (13) and condition (16).

\section{A. Bottom initial state}

Let us first consider the case of the bottom initial state, i.e.,

$$
q_{i}=q_{b}, \quad \dot{q}_{i}=0 .
$$

Before presenting the numerical results, we derive some important general features of the MPEPs and $S_{\min }(t)$. We show in the following that, as the boundary moves from the close vicinity of the bottom toward the saddle, $J(t)$ undergoes qualitative changes while still being stepwise.

First, consider the case when the absorbing wall is close to the bottom: $U(q)$ may then be approximated by a parabola [Fig. 2(a)]

$$
U(q)-U\left(q_{b}\right) \approx \frac{\omega_{0}^{2}}{2}\left(q-q_{b}\right)^{2},
$$

where $\omega_{0}=\sqrt{2}$ and $q_{b}=-1$, in the case of $U(q)$ (3). Thus (15) reduces to a linear equation with constant coefficients that can be integrated explicitly. $S_{\min }(t)$ can be found explicitly too. Rather than presenting some cumbersome formulas we discuss their most important consequence: If $\Gamma<2 \omega_{0}$, then $S_{\min }(t)$ has a stepwise shape [Fig. 2(b)], i.e., possesses inflection points with $d S_{\min } / d t=0$ at

$$
\begin{aligned}
& t=t_{n} \equiv \frac{n \pi}{\omega_{0} \sqrt{1-\left(\Gamma / 2 \omega_{0}\right)^{2}}}, \\
& S\left(t_{n}\right)=\frac{\Delta U_{p}}{1-\exp \left(-\Gamma t_{n}\right)}, \\
& \Delta U_{p} \equiv \omega_{0}^{2}\left(q_{\text {aw }}-q_{b}\right)^{2} / 2, \quad \Gamma<2 \omega_{0}, \quad n=1,2,3, \ldots .
\end{aligned}
$$

The flux barely changes near $t_{n}$ whereas it rises sharply beyond this range provided the corresponding $n$ is not too $\operatorname{large}^{25}$ [Fig. 2(b)]. In the underdamped case, the "length" of each step, $t_{n+1}-t_{n}$, is half a period of eigenoscillation and the "height" of the first steps is large: $S\left(t_{n}\right)-S\left(t_{n+1}\right)$ $\approx \Delta U_{p} \omega_{0} /(\pi \Gamma n(n+1)) \stackrel{\Gamma \rightarrow 0}{\longrightarrow} \infty$. As $\Gamma$ grows, the length of a step increases while the height decreases and, at $\Gamma$ $=2 \omega_{0}$, the steps vanish.

The instants $t_{n}$ mark intervals corresponding to different topologies of the MPEP: For $t \leqslant t_{1},[q(\tau)]$ is monotonic while, for $t_{n}<t \leqslant t_{n+1}(n=1,2,3, \ldots),[q(\tau)]$ possesses $n$ turning points. As $t$ changes, the MPEP varies continuously for any $t$, including $t=t_{n}$. The exit velocity is nonzero unless $t=t_{n}$ [Fig. 2(a)]. 

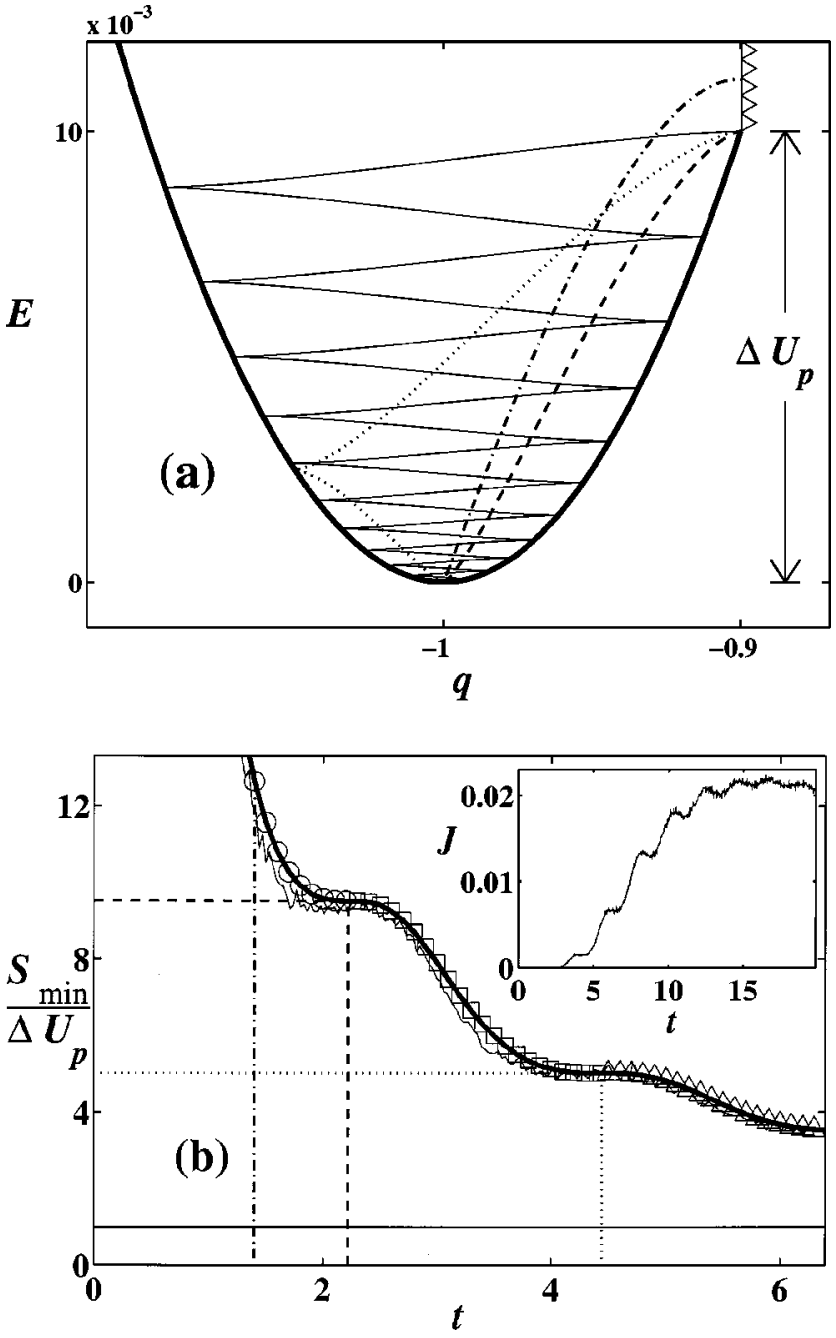

FIG. 2. The case of the bottom initial state. (a) The parabolic approximation $U_{p}(q) \equiv(q+1)^{2}$ (thick solid line) of $U(q)-U\left(q_{b}\right)$ near the bottom, and examples of MPEPs [plotted in the energy-coordinate plane $E-q$ where $E$ $\left.\equiv \dot{q}^{2} / 2+U_{p}(q)\right]$ at $\Gamma=0.05$; the absorbing wall (at $q_{\mathrm{aw}}=q_{\mathrm{aw}}^{(1)} \equiv-0.9$ ) is indicated by triangles; (b) $S_{\min }(t) / \Delta U_{p}$ explicitly calculated in the parabolic approximation is shown by the solid line with markers: circles, squares, and triangles indicate regions corresponding to, respectively, zero, one, and two turning points in the MPEP; $S_{\min }(t) / \Delta U$ derived from simulations in $U(q)$ (3) is shown by the jagged line $\left[\Delta U \equiv U\left(q_{\mathrm{aw}}\right)-U\left(q_{b}\right)\right]$. Dashed and dotted lines indicate the theoretical first and second inflection points with $d S_{\min } / d t=0$, in (b), and the corresponding MPEPs, in (a). The thin solid line shows the large-time asymptote level $(=1)$, in (b), and the corresponding MPEP (which is the time reversal of the noise-free trajectory from the state $\left[q=q_{\text {aw }}, \dot{q}=0\right.$ )], in (a). The dash-dotted line shows in (a) the MPEP for some arbitrarily chosen time $t=1.4$ [see (b)]: It demonstrates that the exit velocity is typically nonzero. The inset shows $J(t)$ measured at $T$ $=\Delta U$.

Apart from a quantitative description of the case when the wall is close to the bottom of the well, the parabolic approximation provides qualitative estimates of the time and energy scales of the steps in the general case. However, some features of the steps $S_{\min }(t)$ and of the associated evolution of the MPEP change qualitatively as the absorbing wall moves toward the saddle.

Let us move the absorbing wall $q_{\text {aw }}$ to a distinctly nonparabolic region of $U(q)$, but still not too close to the saddle $\left(<q_{\mathrm{aw}}^{(c)}\right)$. One can reduce the fourth-order differential equa- tion (15) to a second-order equation for $q$ plus a first-order one for the auxiliary variable $\Gamma^{\prime}:^{21}$

$$
\begin{aligned}
& \ddot{q}+\Gamma^{\prime} \dot{q}+d U / d q=0, \\
& {\left[\Gamma^{\prime}+\left(\Gamma^{2}-\left(\Gamma^{\prime}\right)^{2}\right) / 2\right] \dot{q}^{2}=2 \Gamma \widetilde{E},}
\end{aligned}
$$

where

$$
\widetilde{E} \equiv-\frac{\partial S}{\partial t}=-L+\left(\frac{\partial L}{\partial \dot{q}}-\frac{d}{d t}\left(\frac{\partial L}{\partial \ddot{q}}\right)\right) \dot{q}+\frac{\partial L}{\partial \ddot{q}} \ddot{q}
$$

is conserved along the MPEP, ${ }^{24,21}$ analogously to energy in mechanics. ${ }^{26}$ Given that the initial state is at the bottom, it can be shown that $\widetilde{E} \geqslant 0$ on the MPEP. Allowing for the fact that $\partial S / \partial \dot{q}(t)=0$ on the MPEP,

$$
\frac{d S_{\min }}{d t}=-\left.\widetilde{E}\right|_{\mathrm{MPEP}} \leqslant 0 \text {. }
$$

The system (20), in addition to providing an algorithm ${ }^{27}$ that is faster in some ranges of parameters than solving Eq. (15), has a remarkable feature: If $\widetilde{E}=0$, the equation for $\Gamma^{\prime}$ can be integrated explicitly. ${ }^{21}$ So, the fourth-order equation (15) reduces to a closed second-order equation. ${ }^{28}$ Allowing for $\dot{q}_{i}=0$, the equation for the time-reversed trajectory $[\tilde{q}(\tau)] \equiv[q(t-\tau)]$ becomes [for the sake of convenience, we also present in Eq. (23) the initial $\tilde{q}$ which follows from the third of conditions (13)]

$$
\begin{aligned}
& \frac{d^{2} \tilde{q}}{d \tau^{2}}+\Gamma \frac{1+A e^{\Gamma \tau}}{1-A e^{\Gamma \tau}} \frac{d \tilde{q}}{d \tau}+\frac{d U(\tilde{q})}{d \tilde{q}}=0, \quad A=e^{-\Gamma t}, \\
& \tilde{q}(0)=q_{\text {aw }} .
\end{aligned}
$$

The derivative $d \tilde{q}(\tau=0) / d \tau$ must be chosen such that the condition (16) is satisfied: comparing Eq. (23) at $\tau=0$ with Eq. (16), we come to the important conclusion that

$$
d \tilde{q}(\tau=0) / d \tau=0,
$$

i.e., the MPEP has a zero exit velocity if $d S_{\min } / d t=0$.

One can show (cf. Ref. 21) that the number of possible finite values of $t$ in Eq. (23), such that $\tilde{q}(t)=q_{b}$, equals the number $N$ of turning points in the noise-free $(t=\infty)$ trajectory. Labeling such times $t$ as $t_{n} \equiv t_{n}\left(q_{\text {aw }}\right)(n=1,2, \ldots, N)$, one may relate $n$ to the number $n_{\text {tp }}$ of turning points in the trajectory (23) and (24): $n=n_{\mathrm{tp}}+1 . t_{n}$ increases with $n$ and, if $N=\infty$, the trajectory (23) and (24) for $t=t_{n}$ with $n \rightarrow \infty$ coincides with the noise-free trajectory. If

$$
\Gamma<2 \omega_{0},
$$

then $N=\infty^{26,21}$ while, if $\Gamma \geqslant 2 \omega_{0}$, then typically $N=0$. In rare cases, there is a finite $N \neq 0$ at $\Gamma \geqslant 2 \omega_{0} \cdot{ }^{21}$

Thus, if $\Gamma<2 \omega_{0}$, then $S_{\min }$ decreases with $t$ monotonically, possessing an infinite number of inflection points $t_{n}$ with $d S_{\min }\left(t_{n}\right) / d t_{n}=0$ [Fig. 3(a)]. They divide the time axis into intervals where the MPEP has different numbers of turning points: As $t$ increases, the transformation of the MPEP with $n-1$ turning points, into one with $n$ points, occurs continuously at $t=t_{n}$. 

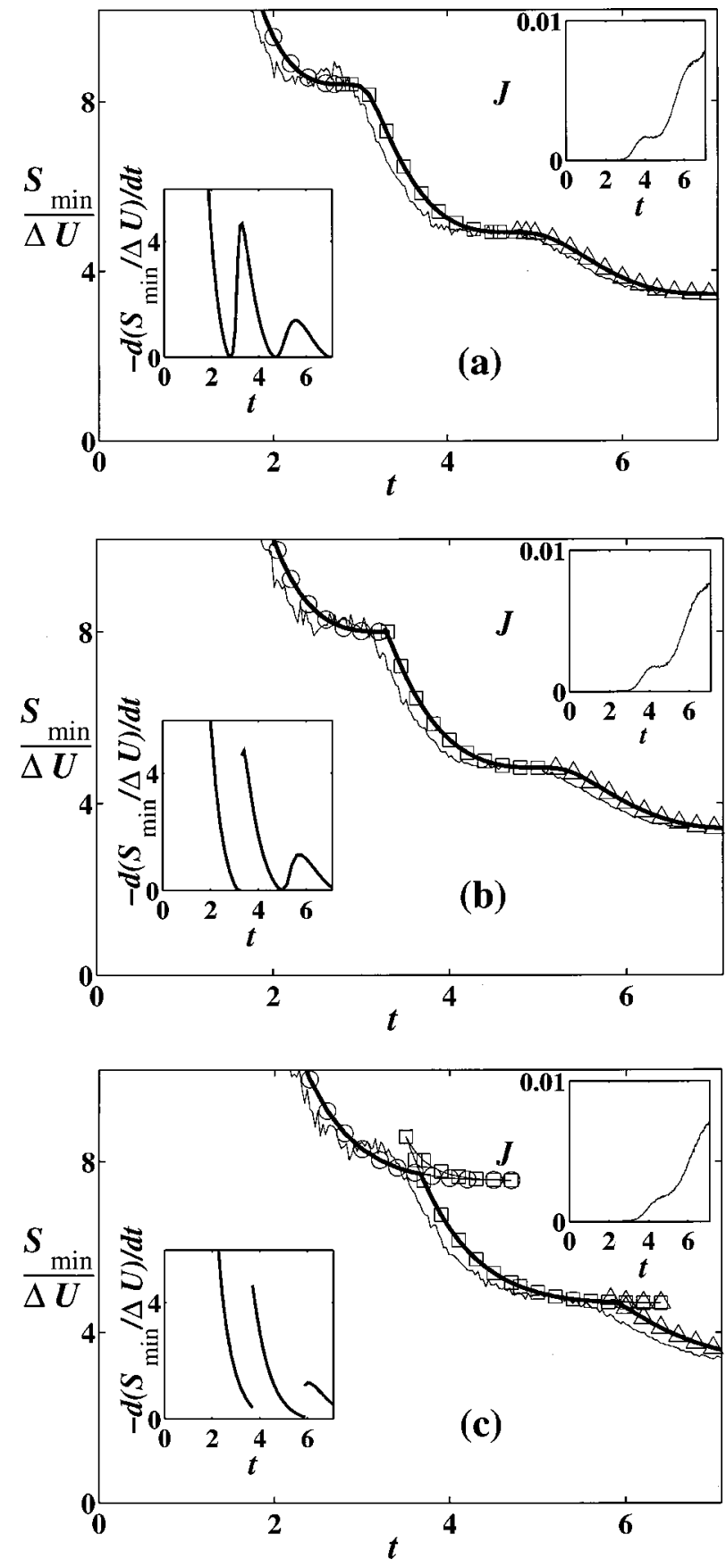

FIG. 3. The case of the bottom initial state. The evolution of $S_{\min }(t)$ normalized by $\Delta U \equiv U\left(q_{\text {aw }}\right)-U\left(q_{b}\right)$ (thick and jagged lines for the theory and simulations, respectively) as $q_{\mathrm{aw}}$ increases: (a) $q_{\mathrm{aw}}=q_{\mathrm{aw}}^{(2)} \equiv 0$, (b) $q_{\mathrm{aw}}$ $=0.371 \approx q_{\mathrm{aw}}^{(c)}$, (c) $q_{\mathrm{aw}}=q_{\mathrm{aw}}^{(3)} \equiv 0.9 . \Gamma=0.05$. Branches of $S(t)$ corresponding to zero, one, or two turning points in the escape path are shown by thin lines marked by circles, squares, or triangles, respectively: In (a) and (b), only one branch exists at each $t$ while, in (c), a few branches coexist in some ranges of $t$ [activation energy $S_{\min }(t)$ coincides with the lowest $S(t)$ ]. Left and right insets show, respectively, $-d\left(S_{\min }(t) / \Delta U\right) / d t$ (theory) and $J(t)$ measured at $T=\Delta U$.

At $\tau=0$, Eq. (23) coincides with the conventional relaxational equation with a finite friction parameter, $\Gamma \operatorname{cth}(\Gamma t / 2)$. Hence, the closer $q_{\text {aw }}$ is to the saddle, the slower the motion near the wall. Thus, $t_{n} \rightarrow \infty$ if $q_{\mathrm{aw}} \rightarrow q_{s}$. On the contrary, the time of motion along MPEPs which get to the wall with nonzero velocity [they relate to sections $S_{\min }(t)$ with nonzero $\left.d S_{\min } / d t\right]$ is less sensitive to the distance $q_{s}-q_{\text {aw }}$ and remains finite even if $q_{\text {aw }}=q_{s}$. Consequently, as $q_{\text {aw }}$ grows, the onset of the fold at $t \approx t_{1}$ (according to numerical calculations) occurs at the critical value $q_{\mathrm{aw}}^{(c)}: d S_{\min } / d t$ is discontinuous at the fold [Fig. 3(b)]. At $q_{\mathrm{aw}}>q_{\mathrm{aw}}^{(c)}$, there are intervals of $t$ during which the system (13), (15)-(17) possesses more than one solution, ${ }^{29}$ i.e., $S(t)$ satisfying (13), (15)-(17) has a multibranch structure [Fig. 3(c)]. Moreover, the closer $q_{\text {aw }}$ is to $q_{s}$, the larger is the number of such intervals and the maximal possible number of coexisting solutions. In order to find the activation energy at a given $t$ one should choose from the solutions of (13), (15)-(17) the minimal one. There are switches between different branches at certain critical times. These can be compared to switching processes, as other parameters vary, in certain escape problems; $;^{22,21,18}$ see also Sec. III. The switches result in jumpwise changes of the MPEP while the activation energy still remains continuous [Fig. 3(c)]. At the same time, the switch results in a discontinuity $d S_{\min } / d t$ : Its values on different sides of the fold differ drastically, so that $S_{\min }(t)$ and $J(t)$ are still distinctly stepwise (stair-like).

We have tested some of the above-mentioned predictions using computer simulations. $S_{\min }(t)$ is derived via optimal fitting of $J(t)$ obtained at different $T$. Figures 2(b) and 3 show reasonable agreement between $S_{\min }(t)$ from the theory and from the simulations. The growth of the flux is clearly stepwise (see insets) in both cases.

\section{B. Nonbottom initial state with a given coordinate and velocity}

If the initial state with a given coordinate and velocity, $\left\{q_{i}, \dot{q}_{i}\right\}$, is shifted from the bottom of the well $\left\{q_{b}, 0\right\}$ then $S_{\min }(t)$ changes: cf. Fig. 4. In particular, $S_{\min }(t)$ becomes nonmonotonic. Moreover, as is evident in Fig. 4, even a tiny shift of the energy from the bottom results in quite a significant distortion of $S_{\min }(t)$ : The shift of energy in Fig. 4(a) and 4(b) is equal to $\Delta U_{p} / 100$ and $\Delta U_{p} / 200$, respectively. Such strong sensitivity to the initial state can be explained by the singularity in the effective time-dependent damping parameter in Eq. (23), which describes the MPEP; so, the shift in the activation energy depends nonanalytically on the shift of the energy of the initial state.

\section{Thermalized initial state}

A nonbottom initial state with a given coordinate and velocity might seem a rather artificial situation but, at the same time, there is always some nonzero initial temperature $T_{0}$ so that various nonbottom states are necessarily involved. The strong sensitivity of the flux $J_{q_{i}, \dot{q}_{i}}(t)$ to the shift of $\left\{q_{i}, \dot{q}_{i}\right\}$ from the bottom, appears to cast doubt on the generality of the stepwise growth $J$ in real situations. However, a rigorous analysis (see the following) shows that the flux at short time scales still grows in a stepwise manner for any temperature $T_{0}<T$. Moreover, if $T_{0} / T \ll \Gamma / \omega_{0}$, then the stepwise structure for flux growth is similar to that obtained using the bottom as the initial state.

So, let the distribution of initial coordinates and velocities be quasistationary for some low temperature $T_{0}$ : 

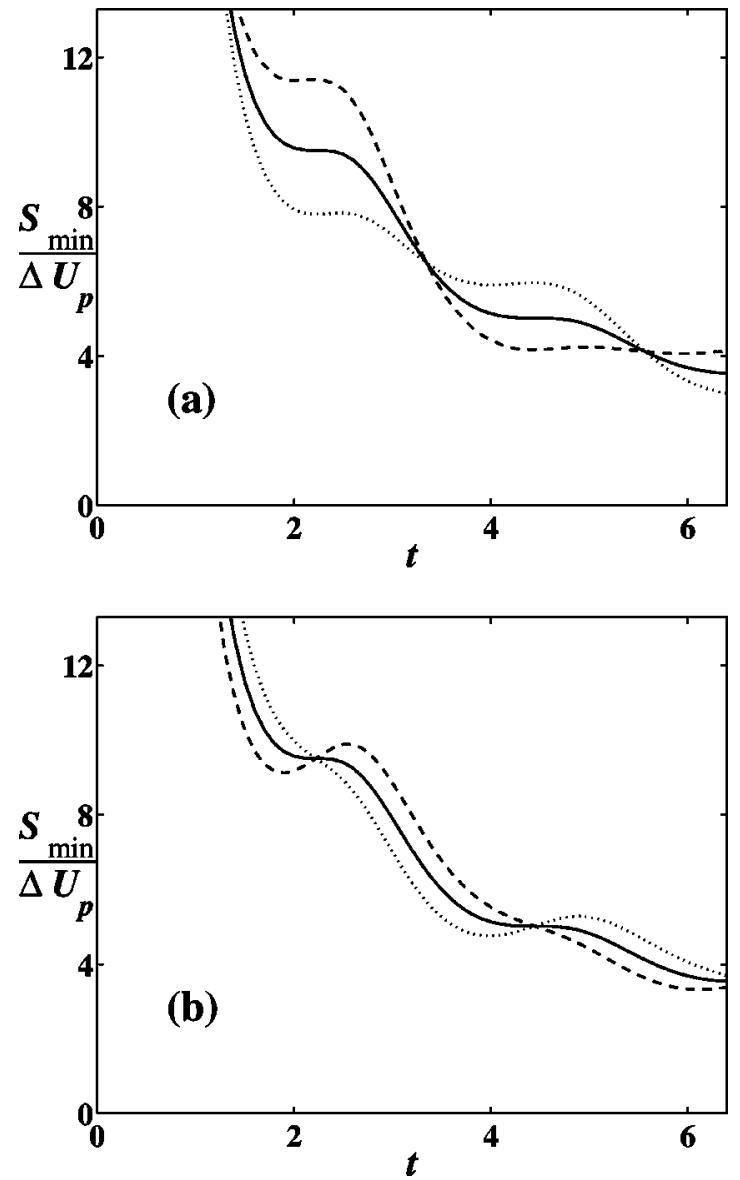

FIG. 4. Comparison between $S_{\min }(t)$ for the bottom initial state (solid line) and for two other initial states with given coordinate and velocity close to those in the bottom, with all other parameters the same as in Fig. 2: (a) $\dot{q}_{0}$ $=0$ while $q_{0}=q_{b}-0.01$ (dotted line) or $q_{0}=q_{b}+0.01$ (dashed line); and (b) $q_{0}=q_{b}$ while $\dot{q}_{0}=-0.01$ (dotted line) or $\dot{q}_{0}=0.01$ (dashed line).

$$
\begin{aligned}
W_{\text {in }}\left(q_{0}, \dot{q}_{0}\right) & =W_{\mathrm{qs}}\left(q_{0}, \dot{q}_{0}\right) \\
& \approx\left\{\begin{array}{l}
Z^{-1} \exp \left(-E_{0} / T_{0}\right) \text { for } E_{0}<U\left(q_{\mathrm{aw}}\right), \\
0 \quad \text { for } E_{0}>U\left(q_{\mathrm{aw}}\right)
\end{array}\right.
\end{aligned}
$$

$$
\begin{aligned}
& E_{0} \equiv \dot{q}_{0}^{2} / 2+U\left(q_{0}\right), \\
& Z=\iint_{E_{0}<U\left(q_{\mathrm{aw}}\right)} d q_{0} d \dot{q}_{0} \exp \left(-E_{0} / T_{0}\right) .
\end{aligned}
$$

We assume that the probability for the system to leave the well before the relevant "initial" instant $t=0$ is negligible.

If at the "initial" instant $t=0$ the additional noise source is switched on, so that the effective temperature becomes $T$ $>T_{0},{ }^{30}$ the evolution of the flux (6) with the initial distribution (26) becomes relevant. Given the activationlike structure of $J_{q_{0}, \dot{q}_{0}}(t)$ [Eqs. (10)-(13)], the flux with the thermalized initial state can be presented in the form

$$
J(t) \equiv J_{T_{0}}(t)=\widetilde{P} \exp \left(-\frac{\widetilde{S}_{\min }(t)}{T}\right),
$$
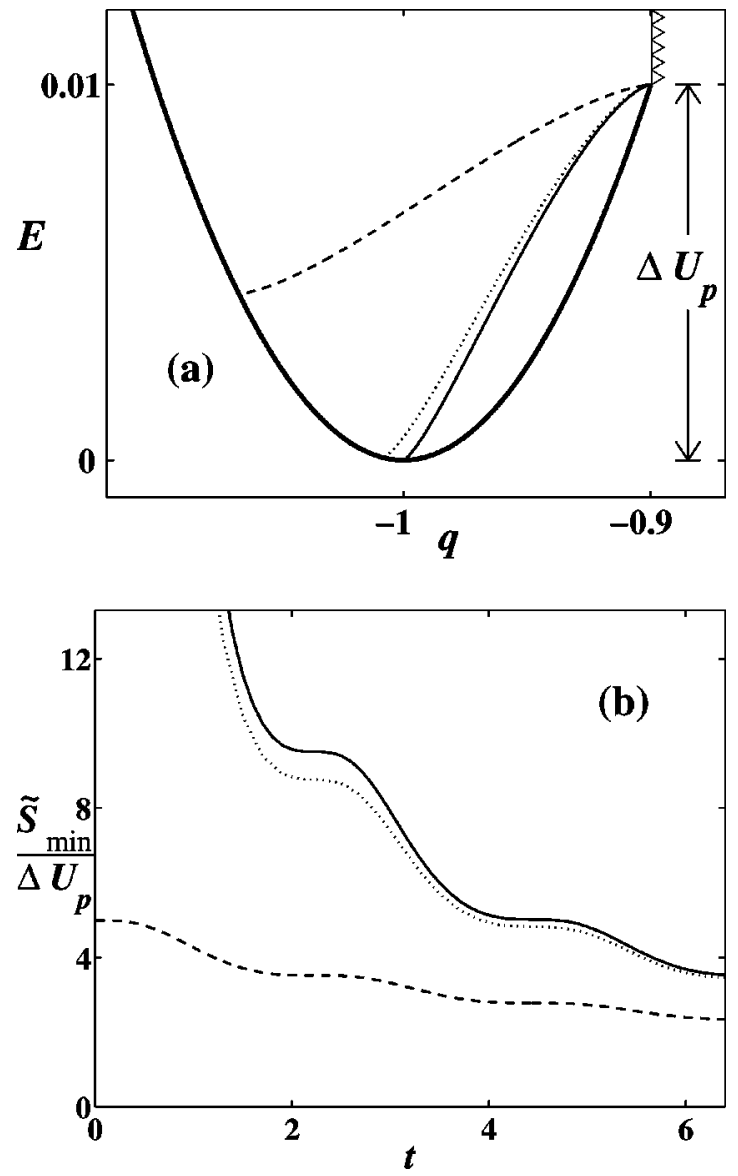

FIG. 5. The case of the thermalized initial state. (a) MPEPs for $t=2.222$, for three characteristic values of $T_{0} / T$, with all other parameters the same as in Fig. 2: $T_{0} / T=0$ (solid line), 0.01 (dotted line), 0.2 (dashed line); (b) $\widetilde{S}_{\min }(t)$ for $T_{0} / T=0$ (solid line), 0.01 (dotted line), 0.2 (dashed line).

where $\widetilde{P}$ is some prefactor and $\widetilde{S}_{\min }$ is the generalized activation energy:

$$
\widetilde{S}_{\min } \equiv \widetilde{S}_{\min }\left(\frac{T_{0}}{T}, t\right)=\min _{q_{0}, \dot{q}_{0}}\left\{S_{\min }\left(q_{0}, \dot{q}_{0}, t\right)+\frac{T}{T_{0}} E_{0}\right\},
$$

where $S_{\min }\left(q_{0}, \dot{q}_{0}, t\right)$ is given by (11)-(13) and $E_{0}$ is defined in (26).

There is no room here to provide details but it can be shown that, for any $T_{0}<T$, the function $\widetilde{S}_{\min }\left(T_{0} / T, t\right)$ is stepwise in $t$. Analogously to the case of the bottom initial state, $\widetilde{S}_{\min }$ possesses inflection points with $d \widetilde{S}_{\min } / d t=0$, provided the wall is not too close to the saddle, and the corresponding MPEPs are described by an equation similar to (23) but with the constant $A$ related to $t$ as

$$
A=e^{-\Gamma t}\left(1-\frac{T_{0}}{T}\right)
$$

The relevant instants $t$ are determined using the condition $\dot{\tilde{q}}(t)=0$ (unlike the condition $\tilde{q}(t)=q_{b}$ relevant to the bottom initial state). 

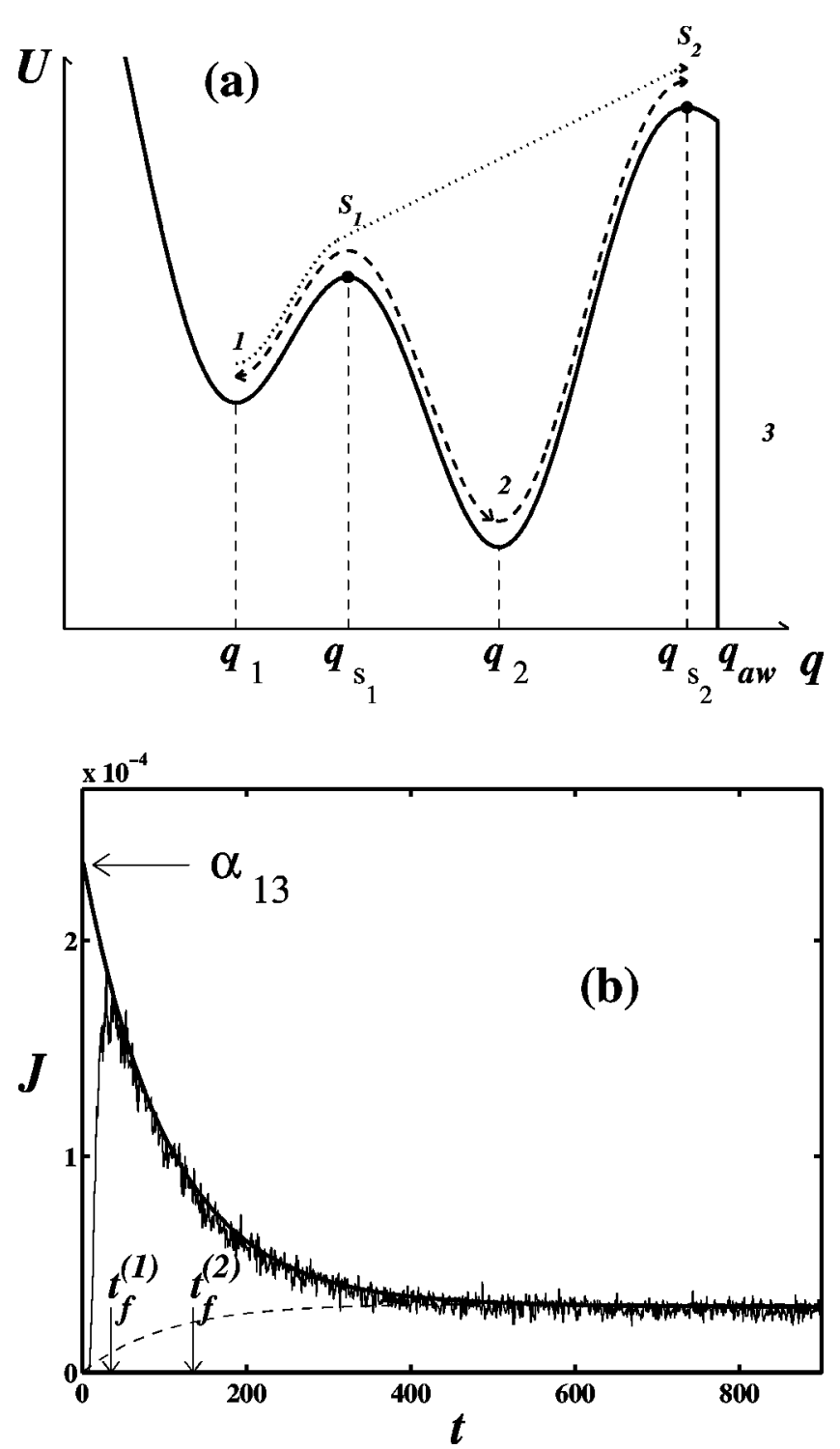

FIG. 6. (a) The potential (4) and a sketch of direct (dotted line) and indirect (dashed line) escape paths $1 \rightarrow s_{2}$; thin dashed lines indicate positions of the local minima $\left(q_{1}, q_{2}\right)$ and maxima $\left(q_{\mathrm{s}_{1}}, q_{\mathrm{s}_{2}}\right)$; (b) simulations of the dependence of the escape flux on time $J(t)$ (thin line) for the model (1), (4) at $\Gamma$ $=0.15, T=0.4$. The thick full and dashed lines show the approximation of $J(t)$ by Eq. (31) in which $\alpha_{12}, \alpha_{21}, \alpha_{\mathrm{qs}}$ are calculated by the KramersMelnikov formula (Ref. 4). For the thick full line, $\alpha_{13,23}=\alpha_{\mathrm{qs}}(1$ $\left.+\left\{\Omega_{1} \Omega_{2}^{-1} \exp \left[\left(U_{1}-U_{2}\right) / T\right]\right\}^{ \pm 1}\right) /\left(1+\left\{m \exp \left[k S_{\min }\left(s_{2} \rightarrow s_{1}\right) / T\right]\right\}^{ \pm 1}\right) \quad$ where $\Omega_{1,2}$ are the frequencies of eigenoscillation in the bottom of wells 1,2 , respectively, $k$ is equal to $1,-1$ for the ranges $\Gamma$ providing $s_{2} \rightarrow 2,1$ respectively, $S_{\min }\left(s_{2} \rightarrow s_{1}\right)$ is calculated from the theory (Ref. 21), and $m$ is the only adjustable parameter ( $m \approx 1.1$ for these parameters); for the dashed line, $\alpha_{13}=0$ and $\alpha_{23}=\alpha_{\mathrm{qs}}\left(1+\alpha_{21} / \alpha_{12}\right)$.

It can be shown that $\widetilde{S}_{\min }\left(T_{0} / T, t \sim \omega_{0}^{-1}\right)$ is close to $S_{\min }\left(q_{i}=q_{b}, \dot{q}_{i}=0, t \sim \omega_{0}^{-1}\right)$ provided

$$
\frac{T_{0}}{T} \ll \frac{\Gamma}{\omega_{0}} .
$$

Otherwise $\widetilde{S}_{\min }\left(t \sim \omega_{0}^{-1}\right)$ is significantly lower and the steps are smeared [Fig. 5(b)].

The competition between the two small parameters, $T_{0} / T$ and $\Gamma / \omega_{0}$, is readily interpreted physically. On one hand, the escape flux (on $t \sim \omega_{0}^{-1}$ ) from the bottom is $\propto \exp \left(-a \Delta U /\left(T \Gamma / \omega_{0}\right)\right)$ where $a \equiv a(t) \sim 1$. On the other hand, if the system starts its motion from an energy $E_{0}$ close to the barrier level, the probability of escape for time $t \sim \omega_{0}^{(-1)}$ will be $\sim 1$, but then the probability to have such starting energy is $\propto \exp \left(-\Delta U / T_{0}\right)$. It is the competition between these two exponentially weak processes which leads to the relation (30). Figure 5(a) shows that, for $T_{0} / T=0.01 \ll \Gamma / \omega_{0}$ $\approx 0.035$, the MPEP starts close to the bottom while, for $T_{0} / T=0.2 \gg \Gamma / \omega_{0}$, the starting energy is $\sim \Delta U$.

\section{MULTIWELL METASTABLE POTENTIAL}

As an example of the multiwell case, we consider the potential (4), which describes the simplest SQUID. ${ }^{2} \mathrm{We}$ place an absorbing wall ${ }^{8}$ at $q_{\text {aw }}=4.5$ [Fig. 6(a)] while the initial state of the system (1), (4) may be any state within well 1; in simulations, we put it at the bottom of well 1 , for the sake of simplicity. We emphasize also that the type of the boundary is not important either, e.g., our results are equally valid for the transition rates between nonadjacent wells in the stable potential with more than two wells. ${ }^{21}$

Unlike the single-well case, where the formation time of quasiequilibrium is of the order of $t_{f}^{(s)}(7)$, its formation in the multiwell case proceeds via two distinct stages: first, quasiequilibrium is formed within the initial well, which takes $t_{f}^{(1)} \sim t_{f}^{(s)}: J$ evolves at this stage quite similarly ${ }^{31}$ to the single-well case; second, quasiequilibrium between wells becomes established, which takes exponentially longer: $t_{f}^{(2)}$ $\sim t_{f}^{(s)} \exp (\Delta U / T) \gg t_{f}^{(1)}$ where $\Delta U$ means a minimal internal barrier. During the latter stage, and during the subsequent quasistationary one, the flux $J(t)$ can be described via a solution of kinetic equations for the well populations, $W_{1}$ and $W_{2}$, using the concept of constant interattractor ${ }^{32}$ transition $^{2}$ rates $\alpha_{i j}$ (cf. Ref. 33):

$$
\begin{aligned}
& J(t) \equiv W_{1} \alpha_{13}+W_{2} \alpha_{23} \\
& \quad=\alpha_{13} e^{-t / t_{f}^{(2)}}+\alpha_{\mathrm{qs}}\left(e^{-t / t_{\mathrm{qs}}}-e^{-t / t_{f}^{(2)}}\right), \\
& t_{f}^{(2)} \approx \alpha_{12}^{-1}, \quad t_{\mathrm{qs}} \approx \alpha_{\mathrm{qs}}^{-1} \approx \alpha_{12} /\left(\alpha_{12} \alpha_{23}+\alpha_{21} \alpha_{13}\right), \\
& T \ll U_{s_{1}}-U_{1}, \quad t \gg t_{f}^{(1)} .
\end{aligned}
$$

The physical meaning of the two terms in (31) is easily understood (cf. Fig. 6). The first one corresponds to direct escapes, i.e., those that do not go via the bottom of well 2, and it dominates until quasiequilibrium becomes established. The second term, corresponding to indirect escapes, i.e., those that involve one or more intermediate transitions between wells 1 and 2 while the ultimate transition to 3 may occur from either well. It dominates during the ensuing quasistationary stage: It is the asymptotic part of this latter flux, $\alpha_{\mathrm{qs}} \exp \left(-t / t_{\mathrm{qs}}\right)$, that is called the quasistationary flux.

Thus, in order to know the flux dynamics one needs to find the interwell transition rates $\alpha_{i j}$. The rates $\alpha_{12}, \alpha_{21}$ and the quasistationary rate $\alpha_{\mathrm{qs}}$ can be calculated from the Kramers-Melnikov formula. ${ }^{4}$ Thus, only one of the two coefficients $\alpha_{13}$ and $\alpha_{23}$ needs to be found independently. We choose $\alpha_{13}$ as the independent coefficient. 


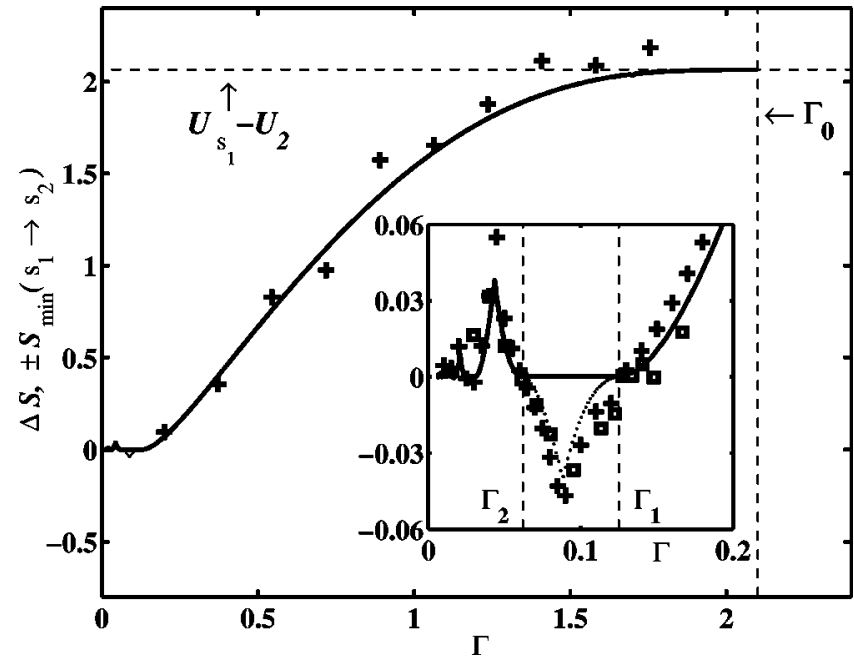

FIG. 7. Theoretical and experimental data on direct escapes/transitions in the metastable potential (4) [Fig. 6(a)]. The calculated excess of action over a difference of energies, $\Delta S\left(1 \rightarrow s_{2}\right)$ (34), is shown by the full line. It is related to the escape rate $\alpha_{13}$. The calculated $\pm S_{\min }\left(s_{2} \rightarrow s_{1}\right)$, related to $R$ (37) by Eq. (38), is shown by the dotted line. It overlaps the full line in the half-plane of positive ordinates. The corresponding quantity (39) based on data obtained by electronic and computer simulations is shown by squares and crosses, respectively. Values of $\Gamma_{n \geqslant 1}$ correspond to noise-free saddle connections with $n-1$ turning points. At $\Gamma=\Gamma_{0}=2 \Omega_{2} \approx 2.1$, the turning points in the noise-free trajectories $s_{2} \stackrel{\text { nf }}{\rightarrow}$ and $s_{1} \rightarrow 2$ disappear. The inset shows the low $\Gamma$ range enlarged.

The theoretical problem of finding $\alpha_{13}$ is inherently difficult. Melnikov pointed out ${ }^{4}$ that, in the multiwell case, his method is valid only if the barriers levels are equal or at least close to each other (cf., e.g., Refs. 4 and 34), a requirement that is often not satisfied. So, the method of optimal fluctuation (cf. Sec. II) was suggested, ${ }^{21}$ seeking the escape rate in the form

$$
\alpha_{13}=P e^{-S_{\min } / T},
$$

where the action $S_{\min }$ does not depend on $T$ and the dependence of the prefactor $P$ on $T$ is relatively weak.

One can show that $S_{\min }$ is the minimum of a certain functional $^{21}$

$$
\begin{aligned}
& S_{\min } \equiv S_{\min }\left(1 \rightarrow s_{2}\right)=\min _{[q(t)], t_{\mathrm{tr}}}(S), \\
& S \equiv S_{t_{\mathrm{tr}}}[q(t)]=\frac{1}{4 \Gamma} \int_{0}^{t_{\mathrm{tr}}} d t(\ddot{q}+\Gamma \dot{q}+d U / d q)^{2}, \\
& q(0)=q_{1}, \quad \dot{q}(0)=0, \quad q\left(t_{\mathrm{tr}}\right)=q_{s_{2}}, \quad \dot{q}\left(t_{\mathrm{tr}}\right)=0,
\end{aligned}
$$

where the trajectory $[q(t)]$ does not pass through attractor 2 . It can easily be shown that the $t_{\text {tr }}$ yielding $S_{\min }$ is equal to $\infty$. The $[q(t)]$ yielding $S_{\min }$ is called ${ }^{21}$ the most probable direct transition path (MPDTP). The main features of $S_{\min }$ and the MPDTP are illustrated in Figs. 7 and 8 for the system (1) and (4); see Ref. 21 for a rigorous general treatment. ${ }^{35}$

Figure 7 shows how the excess action

$$
\Delta S \equiv \Delta S\left(1 \rightarrow s_{2}\right)=S_{\min }\left(1 \rightarrow s_{2}\right)-\left(U_{s_{2}}-U_{1}\right)
$$
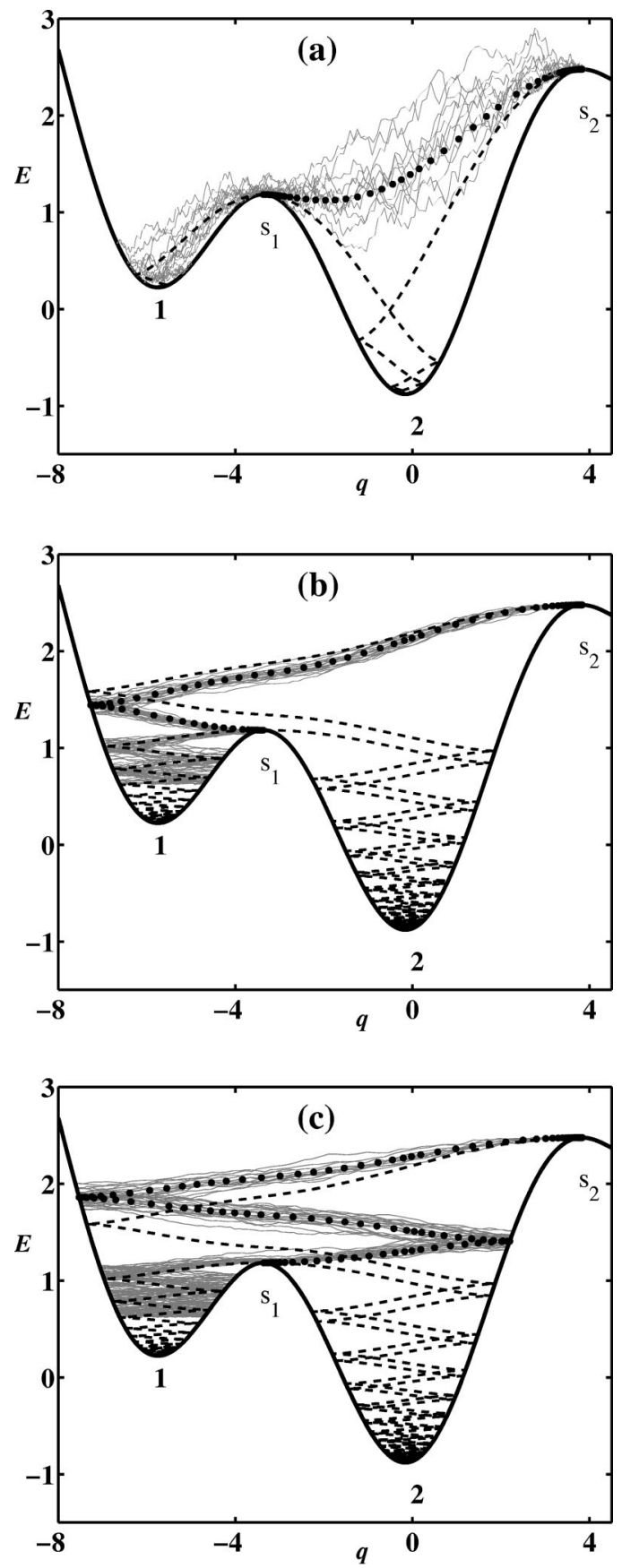

FIG. 8. Simulated direct transition paths $s_{2} \rightarrow 1$ (thin full lines) in the energy-coordinate plane $E-q$ [where $E=\dot{q}^{2} / 2+U(q)$ ] corresponding to (1),(4) at different $\Gamma$ : (a) 0.5 , (b) 0.05 , (c) $0.04[T=0.05$ for (a) and $T$ $=0.005$ for (b), (c)]. The noise-free trajectories $s_{2} \stackrel{\mathrm{nf}}{\rightarrow}$ and $s_{1} \stackrel{\mathrm{nf}}{\rightarrow} 1,2$ are shown by dashed lines. The MPDTPs $s_{2} \rightarrow s_{1}$ are shown by thick dotted lines.

varies with $\Gamma$ over the whole range of $\Gamma$, from very strong damping to the ultraunderdamped case. One can resolve three distinct regions.

The overdamped region can be defined as $\Gamma \geqslant \Gamma_{0}$ $=2 \Omega_{2}$, where $\Omega_{2}$ is the frequency of eigenoscillation in the bottom of well 2 . Here, there is no MPDTP $1 \rightarrow s_{2}$ at all, so that $\alpha_{13}=0$.

In the moderate-friction region, $\left[\Gamma_{1}, \Gamma_{0}\right], \Delta S(\Gamma)$ is monotonic and undergoes its largest variation: from 0 to 
$U_{s_{1}}-U_{2}$. The MPDTP [see Fig. 8(a)] is the time-reversed trajectory $s_{2} \stackrel{A=A_{-}}{\longrightarrow} s_{1} \stackrel{\mathrm{nf}}{\rightarrow} 1$ in which the latter is just the noise-free relaxation from $s_{1}$ to 1 , whereas the former is the solution (cf. Ref. 23 and Sec. II) of

$$
\begin{aligned}
& \ddot{q}_{d}+\Gamma \frac{1+A e^{\Gamma t}}{1-A e^{\Gamma t}} \dot{q}_{d}+d U\left(q_{d}\right) / d q_{d}=0, \\
& q_{d}(0)=q_{s_{2}}, \quad \dot{q}_{d}(0)=0 .
\end{aligned}
$$

Here $A=A_{-}$is a negative constant providing for the path $\left[q_{d}(t)\right]$ to be monotonic [i.e., without turning points]. Note that, in general, there may be an infinite set of $A$ providing $\left[q_{d}(t)\right]$ connecting the saddles: The corresponding trajectories differ by their number of turning points. But $S$ for the other values of $A$ [whose absolute values are smaller than $\left.\left|A_{-}\right|\right]$is larger than that for $A_{-}$.

The underdamped region, $\Gamma \leqslant \Gamma_{1}$, is divided by a number of characteristic values of the friction $\Gamma_{n \geqslant 1}$. Each of these $\Gamma_{n}$ provides for a noise-free saddle connection $\stackrel{\text { nf }}{\rightarrow} s_{1}$, which possesses $n-1$ turning points. In this region, $\Delta S(\Gamma)$ undergoes oscillations corresponding to an alternation between two situations. In the first, $\left[\Gamma_{2 m}, \Gamma_{2 m-1}\right](m$ $\geqslant 1$ ), a noise-free trajectory $s_{2} \stackrel{\mathrm{nf}}{\rightarrow} 1$ exists and the MPDTP is just its time reversal, with $\Delta S=0$. In the second situation, $\left[\Gamma_{2 m+1}, \Gamma_{2 m}\right](m \geqslant 1)$, the action varies nonmonotonically with $\Gamma$, and has cusps. This is due to a competition between the two paths which are the time reversals respectively, of $A_{-} \quad \mathrm{nf} \quad A_{+} \quad \mathrm{nf} \quad A_{ \pm}$ $s_{2} \rightarrow s_{1} \rightarrow 1$ and $s_{2} \rightarrow s_{1} \rightarrow 1$, where $s_{2} \rightarrow s_{1}$ are given by the solutions of (35) with $A_{+} \equiv A_{+}(\Gamma)>0$ and $A_{-} \equiv A_{-}(\Gamma)$ $<0$, respectively: see Fig. 8(b) and 8(c), respectively. As $\Gamma$ varies, $S$ along one path becomes equal to $S$ along another, at a certain $\Gamma$, leading to switching between the paths and to the cusp in $\Delta S(\Gamma)$ : There are corresponding discontinuities in the nonequilibrium potential ${ }^{23}$ and fluctuational separatrix. ${ }^{36}$

Thus, Ref. 21 predicts an exponentially strong dependence of the escape rate $\alpha_{13}$ on friction, including interesting features such as oscillations and cusps, ${ }^{37}$ for $t \gg t_{f}^{(1)}$. To establish whether these, and the properties of MPDTPs described previously, occur in reality, we have undertaken ana$\log$ electronic and computer simulations. A necessary condition is smallness of the temperature: $T \ll \Delta S,\left(U_{s_{1}}\right.$ $\left.-U_{1}\right)$. However to obtain reasonable statistics at such a small temperature would require an unrealistically long time $\left[\propto \exp \left(\left(U_{s_{2}}-U_{1}+\Delta S\right) / T\right)\right]{ }^{38}$ We have overcome this difficulty by exploiting the property of detailed balance, ${ }^{39}$ which implies $^{21}$ that the MPDTP $s_{2} \rightarrow 1$ is just the time reversal of the MPDTP $1 \rightarrow s_{2}$, with the corresponding actions differing by $U_{s_{2}}-U_{1}$, i.e.,

$$
\begin{aligned}
\Delta S\left(1 \rightarrow s_{2}\right) & =S_{\min }\left(s_{2} \rightarrow 1\right) \\
& =\left\{\begin{array}{llll}
0 & \text { at } & s_{2} \rightarrow 1 & \\
& & \text { nf } \\
S_{\min }\left(s_{2} \rightarrow s_{1}\right) & \text { at } & s_{2} \rightarrow 2
\end{array}\right.
\end{aligned}
$$

so that information about the transition $s_{2} \rightarrow 1$ is equivalent to that for $1 \rightarrow s_{2}$, but the experimental time required is of course much smaller in the former case $[\propto \exp (\Delta S / T)]$ than in the latter.

Figure 8(a) demonstrates that, for $\Gamma \in\left[\Gamma_{1}, \Gamma_{0}\right]$, most of the direct paths $s_{2} \rightarrow 1$ do indeed concentrate near $\stackrel{A_{-}}{s_{2} \rightarrow s_{1}} \stackrel{\mathrm{nf}}{\rightarrow} 1$. Figures 8 (b) and 8(c) demonstrate switching of the MPDTP from $s_{2} \rightarrow s_{1} \rightarrow 1$ to $s_{2} \rightarrow s_{1} \rightarrow 1$ as $\Gamma$ decreases in the range $\left[\Gamma_{3}, \Gamma_{2}\right]$.

In order to study $S_{\min }$ we use the following technique. The system is put at $s_{2}$, and one then follows its stochastic dynamics (1), (4) until either the bottom of one of the wells is approached or the coordinate $q_{\text {aw }}$ is reached. After that, the system is reset to $s_{2}$ and the operation is repeated. Once adequate statistics have been obtained, we calculate the ratio of transitions to wells 1 and 2, respectively,

$$
R \equiv R(T)=\frac{N_{s_{2} \rightarrow 1}}{N_{s_{2} \rightarrow 2}} .
$$

It is easy to see that $R \propto \exp \left( \pm S_{\min }\left(s_{2} \rightarrow s_{1}\right) / T\right.$ ) (where + , - correspond to ranges of $\Gamma$ providing $s_{2} \stackrel{\text { nf }}{\rightarrow} 1,2$, respectively). So, $S_{\min }\left(s_{2} \rightarrow s_{1}\right)$ is related to $R$ (37) as

$$
\pm S_{\min }\left(s_{2} \rightarrow s_{1}\right)=\lim _{T \rightarrow 0}[T \ln (R(T))]
$$

where,+- correspond to $s_{2} \rightarrow 1,2$, respectively.

In practice, however, there is always a lower limit for $T$ in simulations, $T_{l}$, because the overall simulation time must not become unrealistically long. That is why the use of (38) may, in practice, introduce significant inaccuracy. To reduce the influence of the preexponential factor we measure $R$ both at $T_{l}$ and at a slightly higher temperature, $T_{l}+\Delta T\left(T_{l} \gg \Delta T\right.$ $\left.\gtrless T_{l}^{2} / S_{\min }\left(s_{2} \rightarrow s_{1}\right)\right)$, so that

$$
\pm S_{\min }\left(s_{2} \rightarrow s_{1}\right) \approx \frac{T_{l}^{2}}{\Delta T} \ln \left(\frac{R\left(T_{l}+\Delta T\right)}{R\left(T_{l}\right)}\right) .
$$

The quantities on the left- and right-hand sides of Eq. (39) are shown in Fig. 7, respectively, by the dotted line (theory) and by squares and crosses (electronic and computer simulations, respectively). The agreement is satisfactory, given that $5 \leqslant S_{\min } / T_{l} \leqslant 7$.

Note that the magnitude of the largest oscillation in action may significantly exceed $U_{s_{2}}-U_{1}$. This occurs if the initial well 1 is adjacent to an external saddle $s_{2}$ while its depth is much less than that of the other well.

Finally, we comment on the experimental consequence of the cutoff of the MPDTP, namely the drastic change of the time evolution of $J$ for $t_{f}^{(1)} \lesssim t \ll t_{f}^{(2)}$ : At $\Gamma<\Gamma_{0}$, one may in principle make $T$ small enough that the sharp growth of $J(t)$ at $t \leqslant t_{f}^{(1)}$ turns into a nearly constant value at $t_{f}^{(1)} \ll t$ $\ll t_{f}^{(1)} \alpha_{13} /\left(\alpha_{12} \alpha_{23}\right)$ while, at $\Gamma>\Gamma_{0}, J(t) \approx \alpha_{12} \alpha_{23} t$ over the whole relevant time-scale: cf. the thin full and dashed lines in Fig. 6(b). Another drastic change occurs with the time evolution of the transition flux $s_{2} \rightarrow 1$ : At $\Gamma<\Gamma_{0}$, it has a 
high narrow peak at $t \approx t_{f}^{(1)}$ whereas, at $\Gamma>\Gamma_{0}$, it is a monotonically and very slowly growing function of $t$, at $t \leqq t_{f}^{(2)}$.

\section{CONCLUSIONS AND OPEN PROBLEMS}

We conclude that: (i) escape from the metastable part of a potential differs markedly after and before the formation of quasiequilibrium within the metastable region; (ii) at time scales much less than the time of the formation of quasiequilibrium within a single well $t_{f}^{(s)}$, the escape flux $J$ grows exponentially strongly with time and, moreover, if the friction $\Gamma$ is small, it does so in a stepwise manner and depends exponentially strongly on $\Gamma$; and (iii) if the metastable part of the potential consists of more than one well, then the formation of quasiequilibrium takes an exponentially long period of time. In the latter case, the escape flux during the most of the period is formed from direct escapes and depends exponentially strongly on friction; moreover, $J(\Gamma)$ undergoes oscillations in the underdamped range and may drop drastically if $\Gamma$ exceeds a critical value $\Gamma_{0} \geqslant 2 \Omega_{2}$.

Open problems yet to be addressed include the following.

(1) For the range $t \ll t_{f}^{(s)}$ : (a) details of the case considered previously, including an accurate study of oscillations of the exit velocity and $d S_{\min } / d t$ with time, as well as of the transition from a smooth $S_{\min }(t)$, with inflection points only, to an $S_{\min }(t)$ possessing folds; (b) additional features characteristic of other types of absorbing boundary, in particular interwell transitions in the multiwell case; (c) a careful consideration of the case with two absorbing walls with the initial state on one of the walls, a case that is relevant, e.g., to ionic channels ${ }^{40}$ and the preliminary analysis indicates oscillations of the flux in time; (d) a generalization for nonpotential systems and/or nonwhite noise for which, unlike potential systems subject to white noise where switching between different MPEPs gives rise only to folds in $S_{\min }(t)$, we anticipate the possibility of jumps in $S_{\min }(t)$.

(2) Both for $t \ll t_{f}^{(s)}$ and for $t_{f}^{(1)} \ll t \leqslant t_{f}^{(2)}$ (the multiwell case only, for the latter range): (a) the prefactor; and (b) multidimensional problems.

\section{ACKNOWLEDGMENTS}

The research has been supported by INTAS Grant No. 97-574, and by the Engineering and Physical Sciences Research Council (UK). We are grateful to George Pickett for his assistance with some of the figures.

${ }^{1}$ H. A. Kramers, Physica (Amsterdam) 7, 284 (1940).

${ }^{2}$ K. K. Likharev, Dynamics of Josephson Junctions and Circuits (Gordon and Breach, Philadelphia, 1986).

${ }^{3}$ P. Hanggi, P. Talkner, and M. Borkovec, Rev. Mod. Phys. 62, 251 (1990).

${ }^{4}$ V. I. Mel'nikov, Phys. Rep. 209, 1 (1991).

${ }^{5}$ A. N. Cleland and M. L. Roukes, Nature (London) 392, 160 (1998).

${ }^{6}$ V. A. Shneidman, Phys. Rev. E 56, 5257 (1997).

${ }^{7}$ E. W. G. Diau et al., Science 279, 847 (1998).

${ }^{8}$ The absorbing wall is equivalent to the barrier falling down to $-\infty$, which is illustrated by Fig. 6(a).

${ }^{9}$ The choice of $t_{l}$ is quite arbitrary: It should exceed the relevant time scale and is introduced purely to reduce the overall time of experiment.

${ }^{10}$ R. P. Feynman and A. R. Hibbs, Quantum Mechanics and Path Integrals (McGraw-Hill, New York, 1965).
${ }^{11}$ M. I. Dykman, P. V. E. McClintock, V. N. Smelyanski, N. D. Stein, and N. G. Stocks, Phys. Rev. Lett. 68, 2718 (1992).

${ }^{12}$ M. Arrayás, I. Kh. Kaufman, D. G. Luchinsky, P. V. E. McClintock, and S. M. Soskin, Phys. Rev. Lett. 84, 2556 (2000).

${ }^{13}$ S. M. Soskin, V. I. Sheka, T. L. Linnik, and R. Mannella, Phys. Rev. Lett. 86, 1665 (2001); ibid. Erratum (to be published).

${ }^{14}$ If the wall is close to the bottom, the estimate (7) can be obtained by consideration of the parabolic potential: The Fokker-Planck equation is then solved explicitly (Ref. 15). It follows directly that, if the initial state corresponds to the bottom of the well, i.e., $W(q, \dot{q}, t=0)=\delta(q$ $\left.-q_{b}\right) \delta(\dot{q})$, then $W$ near the energy level $\Delta U$ and the coordinate $\sqrt{2 \Delta U} / \omega_{0}$ evolves close to the equilibrium value for the time described by Eq. (7). In another characteristic case, when the wall is close to the saddle (cf. $q_{\mathrm{aw}}^{(3)}$ in Fig. 2), the estimate (7) is also typically valid: It agrees with the explicit results (Ref. 6) for the underdamped and overdamped limits provided that the curvatures at the saddle and at the bottom are of the same order, which in fact is typically the case: cf. $U(q)(1)$.

${ }^{15}$ M. C. Wang and G. E. Uhlenbeck, Rev. Mod. Phys. 17, 323 (1945).

${ }^{16}$ In recent papers (Refs. 17-20), the method of optimal fluctuation was applied to problems which, though having some similarity to the nonstationary Kramers problem, are distinctly different: References 17-19 considered nonstationary optimal paths in the overdamped potential system in the context of the prehistory problem (Ref. 11) while Ref. 20 considered, in particular, the time dependence of escape rates in the overdamped system subject to the colored noise.

${ }^{17}$ B. E. Vugmeister et al., Phys. Rev. E 55, 5338 (1997).

${ }^{18}$ R. Mannella, Phys. Rev. E 59, 2479 (1999).

${ }^{19}$ B. E. Vugmeister et al., Phys. Rev. E 59, 2481 (1999).

${ }^{20}$ E. Cortes and F. Espinosa, Physica A 267, 414 (1999).

${ }^{21}$ S. M. Soskin, J. Stat. Phys. 97, 609 (1999).

${ }^{22}$ R. S. Maier and D. L. Stein, J. Stat. Phys. 83, 291 (1996).

${ }^{23}$ R. Graham and T. Tel, Phys. Rev. A 33, 1322 (1986).

${ }^{24}$ L. E. Elsgolc, Calculus of Variations (Pergamon, London, 1961).

${ }^{25}$ If $n \rightarrow \infty$, so that $t_{n} \rightarrow \infty$, then $S\left(t_{n}\right)$ reduces to $\Delta U_{p}$ while the MPEP reduces to the time-reversal of the noise-free relaxational trajectory from the state $\left\{q=q_{\text {aw }}, \dot{q}=0\right\}$, thus recovering the conventional Arrhenius activation energy for the quasistationary flux (Ref. 1) and the corresponding MPEP (Refs. 23 and 21), respectively. The reduction of the asymptotic (at $t \rightarrow \infty)$ activation energy and MPEP to their conventional forms is valid for an arbitrary $U(q)$.

${ }^{26}$ L. D. Landau and E. M. Lifshitz, Mechanics (Pergamon, London, 1976).

${ }^{27}$ To avoid the singularities encountered by $\Gamma^{\prime}$ at the turning points, one may use the variable $G \equiv \Gamma^{\prime} \dot{q}$ instead.

${ }^{28}$ A similar equation was obtained in Ref. 23 but in a different context and by a different method.

${ }^{29}$ The numerical search is more difficult than in the case of a single solution. Details will be presented elsewhere.

${ }^{30}$ Note that the additional noise source does not need necessarily a thermal origin. In SQUIDs, for example, it could be an external magnetic flux noise.

${ }^{31}$ Our qualitative analysis indicates that, in addition, some new features may appear in the multiwell case, but this has not yet been studied in detail.

${ }^{32}$ For the sake of brevity, we refer to region 3 as an "attractor" too.

${ }^{33}$ M. I. Freidlin and A. D. Wentzell, Random Perturbations in Dynamical Systems (Springer, New York, 1984).

${ }^{34}$ R. Ferrando, and R. Spadacini, and G. E. Tommei, Phys. Rev. E 48, 2437 (1993).

${ }^{35}$ Some of the results (Ref. 21) are closely related to some results of Ref. 23 which were obtained by a different method and in a different context.

${ }^{36}$ M. I. Dykman, M. M. Millonas, and V. N. Smelyanskiy, Phys. Lett. A 195, 53 (1994).

${ }^{37}$ In "long" jumps in a multibarrier potential with equal or nearly equal barriers, a monotonic exponential dependence on friction was found in earlier works (see, e.g., Refs. 34 and 4, respectively). However, the obvious reason that oscillations and cusps were not found in those studies is that they can only arise in the case of distinctly different barriers levels.

${ }^{38}$ Note, however that preexponential time scales in other systems (e.g., in SQUIDs) may be much smaller, so that the relevant flux may be readily simulated and/or measured.

${ }^{39}$ H. Risken, The Fokker-Planck Equation, 2nd ed. (Springer, Berlin, 1992).

${ }^{40} \mathrm{~B}$. Hille, Ionic Channels of Excitable Membranes (Sinauer Associates, Sunderland, 1992) 